\title{
Research on Solving Skills of the Second Important Limit Questions in Advanced Mathematics
}

\author{
Jingbo Sun \\ Mathematics and Physics Education Department \\ Yantai Nanshan University \\ Yantai, China
}

\begin{abstract}
The first section of Advanced Mathematics are Functions and Limits and the limits include two important limit formulas of the first important limit and the second important limit which are still valid. In actual process of solving, questions generally are the deformation of the important limit, especially the second important limit. In order to solve them by the formulas, the deformation should be changed to the form of the formulas before calculation, which is complex and easy to make a mistake. Therefore, a simple algorithm is given in this paper by seeking out the relationship between the questions and simplifying the process of transformation.
\end{abstract}

Keywords-important limit; coefficient; denominator; exponent

\section{INTRODUCTION}

The first section of Advanced Mathematics is Function and Limit, and the limit includes two important limit formulas.

The first important limit is $\lim _{x \rightarrow 0} \frac{\sin x}{x}=1$ and the second important limit is $\lim _{x \rightarrow \infty}\left(1+\frac{1}{x}\right)^{x}=\mathrm{e}$ the conclusion is still correct when the $\mathrm{x}$ in the formulas is replaced with a function with the same trend with the $\mathrm{x}$.

\section{ThE METHOD OF CONVENTIONAL EXPRESSION}

In solving the second important limit questions, the result can't be obtained according to the formula calculation until the form of the questions are changed to the form of the formulas. The common transformations are as follows:

\section{Formula}

$$
1
$$$$
\lim _{x \rightarrow \infty}\left(1-\frac{1}{x}\right)^{x}=\lim _{x \rightarrow \infty}\left(1+\frac{1}{-x}\right)^{-x(-1)}=\left[\lim _{x \rightarrow \infty}\left(1+\frac{1}{-x}\right)^{-x}\right]^{-1}=e^{-1} \text {. }
$$

Formula 2 :

$$
\lim _{x \rightarrow 0}(1+x)^{\frac{1}{x}}=\lim _{x \rightarrow 0}\left(1+\frac{1}{\frac{1}{x}}\right)^{\frac{1}{x}}=\mathrm{e}
$$

Formula

$$
3
$$

$\lim _{x \rightarrow \infty}\left(1+\frac{1}{a x}\right)^{x}=\lim _{x \rightarrow \infty}\left(1+\frac{1}{a x}\right)^{\frac{a x}{a}}=\lim _{x \rightarrow \infty}\left[\left(1+\frac{1}{a x}\right)^{\frac{a x}{a}}\right]^{\frac{1}{a}}=\mathrm{e}^{\frac{1}{a}}$

$$
\text { Formula 4: } \lim _{x \rightarrow \infty}\left(1+\frac{\mathrm{b}}{\mathrm{x}}\right)^{x}=\lim _{x \rightarrow \infty}\left[\left(1+\frac{1}{\frac{x}{b}}\right)^{\frac{\mathrm{x}}{b}}\right]^{\mathrm{b}}=\mathrm{e}^{\mathrm{b}}
$$

$$
\begin{aligned}
& \text { Formula } \\
& \lim _{x \rightarrow \infty}\left(1+\frac{\mathrm{c}}{\mathrm{ax}+\mathrm{b}}\right)^{x}=\lim _{x \rightarrow \infty}\left(1+\frac{1}{\frac{a x+b}{c}}\right)=\lim _{x \rightarrow \infty}\left[\left(1+\frac{1}{\frac{a x+b}{c}}\right)^{\frac{\mathrm{ax}+\mathrm{b}}{c}}\right]^{\frac{c}{a}}\left[\left(1+\frac{1}{\frac{a x+b}{c}}\right)^{\frac{-b}{c}}\right]^{\frac{c}{a}} \\
& =e^{\frac{c}{a}}
\end{aligned}
$$

In teaching, after proving the second important limit conclusion, these transformation formulas are given to students to remember the forms and results which can be substituted into similar questions directly to simplify the calculation.

\section{THE METHOD OF OBSERVATION AND SUMMARY}

The phenomenon can be found that no matter what kind of formula, the exponent part of the answer has great relevance with the fraction behind 1 plus in the question, we try to observe through these simple formulas and transformations.

Formula 1: $\lim _{x \rightarrow \infty}\left(1-\frac{1}{x}\right)^{x}=\lim _{x \rightarrow \infty}\left(1+\frac{-1}{x}\right)^{x}=e^{-1}$

Formula 2: $\lim _{x \rightarrow 0}(1+x)^{\frac{1}{x}}=\lim _{x \rightarrow 0}\left(1+\frac{1}{\frac{1}{x}}\right)^{\frac{1}{x}}=\mathrm{e}$

Formula 3 : $\lim _{x \rightarrow \infty}\left(1+\frac{1}{a x}\right)^{x}=\lim _{x \rightarrow \infty}\left(1+\frac{\frac{1}{a}}{x}\right)^{x}=\mathrm{e}^{\frac{1}{a}}$

Formula $4: \lim _{x \rightarrow \infty}\left(1+\frac{\mathrm{b}}{\mathrm{x}}\right)^{x}=\mathrm{e}^{\mathrm{b}}$

Formula 5 :

$$
\lim _{x \rightarrow \infty}\left(1+\frac{\mathrm{c}}{\mathrm{ax}+\mathrm{b}}\right)^{x}=\lim _{x \rightarrow \infty}\left(1+\frac{\frac{c}{a}}{x+\frac{b}{a}}\right)^{x}=e^{\frac{c}{a}}
$$

According to observation, such a conclusion can be made that the exponent part of the answer has great relevance with the fraction behind 1 plus in the question, and it is the numerator of the fraction when the coefficient of $\mathrm{x}$ in denominator of the fraction is 1 . However, it has nothing to do with constant in the denominator. Based on the discovery, it is unnecessary to simplify so many transformations, and it is enough to observe the numerator of the fraction when the denominator of the fraction is 1 . 


\section{EXTENSION AND PROMOTION}

Because it is nothing to do with constant in the denominator, the Formula 5 can be simplified as follows:

$$
\text { Formula } 5: \lim _{x \rightarrow \infty}\left(1+\frac{\mathrm{c}}{\mathrm{ax}+\mathrm{b}}\right)^{x}=\lim _{x \rightarrow \infty}\left(1+\frac{\frac{c}{a}}{x}\right)^{x}=e^{\frac{c}{a}}
$$

Of course, the premise of this conclusion should be paid special attention, is that the exponent part outside bracket must be $x$, if not, it shouldn't be calculated until transformation. So that the complicated second important limit operation can be changed to simple operation, which is verified by a few examples as follows:

Example 1: $\lim _{x \rightarrow \infty}\left(1-\frac{2}{\mathrm{x}}\right)^{x}$

Solution 1: $\lim _{x \rightarrow \infty}\left(1-\frac{2}{\mathrm{x}}\right)^{x}=\lim _{x \rightarrow \infty}\left[\left(1+\frac{1}{-\frac{\mathrm{x}}{2}}\right)^{-\frac{x}{2}}\right]^{-2}=\mathrm{e}^{-2}$

Solution 2: $\lim _{x \rightarrow \infty}\left(1-\frac{2}{x}\right)^{x}=\lim _{x \rightarrow \infty}\left(1+\frac{-2}{x}\right)^{x}=\mathrm{e}^{-2}$

Example 2: $\lim _{x \rightarrow \infty}\left(\frac{x+a}{x-a}\right)^{x}$

Solution

\section{1}

$\lim _{x \rightarrow \infty}\left(\frac{x+a}{x-a}\right)^{x}=\lim _{x \rightarrow \infty}\left(1+\frac{2 \mathrm{a}}{\mathrm{x}}\right)^{x}=\lim _{x \rightarrow \infty}\left[\left(1+\frac{1}{\frac{\mathrm{x}}{2 a}}\right)^{\frac{x}{2 a}}\right]^{2 a}=\mathrm{e}^{2 \mathrm{a}}$

Solution 2: $\lim _{x \rightarrow \infty}\left(\frac{x+a}{x-a}\right)^{x}=\lim _{x \rightarrow \infty}\left(1+\frac{2 \mathrm{a}}{\mathrm{x}}\right)^{x}=\mathrm{e}^{2 \mathrm{a}}$

Example $3: \lim _{x \rightarrow \infty}\left(\frac{x}{1+x}\right)^{x}$

Solution 1

$$
\begin{aligned}
& \lim _{x \rightarrow \infty}\left(\frac{x}{1+x}\right)^{x}=\lim _{x \rightarrow \infty}\left(1-\frac{1}{1+\mathrm{x}}\right)^{x}=\left(1+\frac{1}{-(1+\mathrm{x})}\right)^{-[-(1+x-1)]} \\
& =\left[\left(1+\frac{1}{-(1+\mathrm{x})}\right)^{-(1+x)+1}\right]^{-1}=\mathrm{e}^{-1} \\
& \text { Solution 2: } \lim _{x \rightarrow \infty}\left(\frac{x}{1+x}\right)^{x}=\lim _{x \rightarrow \infty}\left(1+\frac{-1}{1+\mathrm{x}}\right)^{x}=\mathrm{e}^{-1}
\end{aligned}
$$

As mentioned above, it is important to note that the exponent part of the question shall be $\mathrm{x}$, if not, it should be made transformation. In order to prove availability of this method when the exponent part is not $\mathrm{x}$, a few examples are given as follows:

Example $4: \lim _{x \rightarrow \infty}\left(\frac{1+x}{x}\right)^{2 x}$

Solution 1: $\lim _{x \rightarrow \infty}\left(\frac{1+x}{x}\right)^{2 x}=\lim _{x \rightarrow \infty}\left[\left(1+\frac{1}{\mathrm{x}}\right)^{x}\right]^{2}=\mathrm{e}^{2}$
Example 5: $\lim _{x \rightarrow \infty}\left(\frac{2-x}{3-x}\right)^{2+x}$

Solution

$\lim _{x \rightarrow \infty}\left(\frac{2-x}{3-x}\right)^{2+x}=\lim _{x \rightarrow \infty}\left(1+\frac{-1}{3-x}\right)^{x}\left(1+\frac{-1}{3-x}\right)^{2}=\lim _{x \rightarrow \infty}\left(1+\frac{1}{x-3}\right)^{x}\left(1+\frac{1}{x-3}\right)^{2}=\mathrm{e}$

Observing the above examples, we found that this algorithm is the same as general formula algorithm, and is not simplified. However, a new discovery could be found from Example 5 which is that the answer has nothing to do with the constant in the fraction denominator, but also with the constant the exponent. This shows that if the parts have nothing to do with the removed result, the final answer will not be changed, and the whole calculation will become simpler.

Example 5: $\lim _{x \rightarrow \infty}\left(\frac{2-x}{3-x}\right)^{2+x}$

Solution

$\lim _{x \rightarrow \infty}\left(\frac{2-x}{3-x}\right)^{2+x}=\lim _{x \rightarrow \infty}\left(1+\frac{-1}{3-x}\right)^{x}=\lim _{x \rightarrow \infty}\left(1+\frac{1}{x}\right)^{x}=\mathrm{e}$

Example 6 : $\lim _{x \rightarrow \infty}\left(\frac{x+5}{3+x}\right)^{2+3 x}$

Solution

$\lim _{x \rightarrow \infty}\left(\frac{x+5}{3+x}\right)^{2+3 x}=\lim _{x \rightarrow \infty}\left(1+\frac{2}{3+x}\right)^{3 x}=\lim _{x \rightarrow \infty}\left[\left(1+\frac{2}{x}\right)^{x}\right]^{3}=\left(\mathrm{e}^{2}\right)^{3}=\mathrm{e}^{6}$

In conclusion, a general and simple formula algorithm can be obtained:

Formula 6

$\lim _{x \rightarrow \infty}\left(1+\frac{\mathrm{c}}{\mathrm{ax}+\mathrm{b}}\right)^{m x+n}=\lim _{x \rightarrow \infty}\left[\left(1+\frac{\frac{c}{a}}{x}\right)^{x}\right]^{m}=\left(e^{\frac{c}{a}}\right)^{m}=e^{\frac{c m}{a}}$

\section{CONCLUSION}

Since the important limit is a difficult and key problem in Advanced Mathematics teaching, and also is a difficult point which perplexes students in exercises and examinations. With this algorithm, it not only is unnecessary for students to remember so many deformation formulas, but also free them from a large amount of complicated calculation, in the meanwhile improves accuracy and saves time.

\section{REFERENCES}

[1] Write by Department of Mathematics in Tongji University, Advanced Mathematics [M], Higher Education Press, 2012.

[2] $\mathrm{Li} \mathrm{Na}$, Intercommunity between Advanced Algebra and Mathematical Analysis in Some Aspects [J], Science and Technology View, 2014 (33)

[3] Chu Zhiwei, Research on Question-driven Teaching in Mathematical Analysis Teaching [J], Journal of Kaifeng Institute of Education, 2014 (08).

[4] Meng Xianji, Research on Mathematical Analysis Teaching under Computer Algebra System Environment [J], Journal of Shenyang Normal University (Natural Science Edition), 2014 (04). 